\title{
Heat shock protein 27 phosphorylation in the proliferation and apoptosis of human umbilical vein endothelial cells induced by high glucose through the phosphoinositide 3-kinase/Akt and extracellular signal-regulated kinase $1 / 2$ pathways
}

\author{
HAI-FENG CHEN ${ }^{1}$, SHU-JUAN LIU ${ }^{2}$ and GANG CHEN ${ }^{2}$ \\ Departments of ${ }^{1}$ Cardiology and ${ }^{2}$ Endocrinology, Fujian Provincial Clinical College, \\ Fujian Medical University, Fuzhou, Fujian 350001, P.R. China \\ Received November 19, 2013; Accepted September 18, 2014
}

DOI: $10.3892 / \mathrm{mmr} .2014 .2884$

\begin{abstract}
In the present study, the effect of the heat shock protein 27 (HSP27) signaling pathway on the proliferation and apoptosis of human umbilical vein endothelial cells (HUVECs) induced by high glucose (HG) was investigated. HUVEC proliferation in the indicated conditions was measured by the alamarBlue ${ }^{\circledR}$ assay. Apoptosis in HUVECs cultured with $\mathrm{HG}$ was analyzed by an Annexin V-fluorescein isothiocyanate/propidium iodide apoptosis detection kit. HSP27 activity was evaluated by western blotting with specific phospho-HSP27 antibody. HUVEC proliferation induced by $\mathrm{HG}$ was observed to be reduced by the HSP27 inhibitor quercetin in a concentration-dependent manner, with a concomitant increase in apoptosis. The phosphorylation of HSP27 induced by HG was blocked by the specific phosphoinositide 3-kinase (PI3K) inhibitor LY294002 and the specific extracellular signal-regulated kinase (ERK) 1/2 inhibitor U0126 in a concentration-dependent manner, with peak inhibition rates of 62.6 and $56.1 \%$, respectively. LY294002 and U0126 also reduced HUVEC proliferation with a concomitant increase in apoptotic rate. In conclusion, HSP27 phosphorylation is important in mediating the proliferation and apoptosis of HUVECs induced by high glucose, and PI3K/Akt and ERK1/2 are important signaling pathways that contribute to HSP27 phosphorylation.
\end{abstract}

\section{Introduction}

Diabetes mellitus is a serious threat to health and a predominant risk factor in the development of macro- and microvascular

Correspondence to: Professor Hai-Feng Chen, Department of Cardiology, Fujian Provincial Clinical College, Fujian Medical University, 134 Dongjie, Fuzhou, Fujian 350001, P.R. China

E-mail: haifengchencn@163.com

Key words: heat shock protein 27, proliferation, apoptosis, endothelial cells complications. Studies have shown that high glucose (HG) easily induces endothelial cell (EC) injury and apoptosis, which in turn results in diabetic vascular complications (1-4). EC apoptosis has also been closely associated with vascular disease.

Heat shock protein 27 (HSP27) is important in the regulation of proliferation and apoptosis in numerous cell types. Thus, HSP27 may be a potential target for the interventional treatment of pathological processes associated with cell proliferation and apoptosis $(5,6)$. The phosphoinositide 3-kinase (PI3K)-Akt signaling pathway may promote EC proliferative dysfunction in diabetes. The mitogen-activated protein kinase (MAPK)/extracellular signal-regulated kinase (ERK)signaling pathway is involved in proliferation and survival responses in ECs. Several studies (7-15) have shown that the MAPK/ERK and PI3K/Akt signaling pathways regulate the phosphorylation of HSP27. Another previous study has demonstrated that HSP27 phosphorylation is important in mediating the migration of vascular smooth muscle cells (VSMCs), and the MAPK/ERK and PI3K/Akt signaling pathways have also been revealed to regulate the phosphorylation of HSP27 in VSMCs $(16,17)$. In the present study, the aim was to obtain information regarding the signal transduction pathways that regulate HSP27 phosphorylation, proliferation and apoptosis in human umbilical vein ECs (HUVECs) induced by HG.

\section{Materials and methods}

Isolation and culture of human ECs. HUVECs were isolated from the healthy umbilical veins of healthy female patients following laparotomy at the Fujian Provincial Hospital (Fuzhou, Fujian, China). The venous lumen was washed with sterile phosphate-buffered saline (PBS) 2-3 times and pre-warmed collagenase I (13-15 ml) was injected into the lumen, which was incubated for $20 \mathrm{~min}$. The lumen was then washed using $30-40 \mathrm{ml}$ sterile PBS. The wash-out was centrifuged at $155 \mathrm{x} \mathrm{g}$ for $10 \mathrm{~min}$ and the HUVECs were collected subsequent to discarding the supernatant. The cells were cultured in M199 medium with $20 \%$ fetal bovine serum (FBS). The cultures were maintained at $37^{\circ} \mathrm{C}$ with a gas mixture of $5 \% \mathrm{CO}_{2}: 95 \%$ air. The primary cultures had the fluid changed $24 \mathrm{~h}$ after seeding and were 
subcultured on reaching confluence using $0.01 \%$ trypsin-EDTA (Sigma-Aldrich, St. Louis, MO, USA). The ECs were identified by direct immunostaining for factor VIII. The ECs from the third passages in an actively growing condition were used for experiments. The present study was conducted in accordance with the Declaration of Helsinki and with approval from the Ethics Committee of Fujian Provincial Clinical College (Fujian Medical University, Fuzhou, China). Written informed consent was obtained from all patients in this study.

alamarBlue $^{\circledR}$ cell proliferation assay. HUVECs in a good growth condition were inoculated in 96-well plates with 6,000 cells/well and $150 \mu \mathrm{l}$ cell culture medium was added to each well. The day following inoculation, the culture medium was changed to $60 \mu \mathrm{l}$ M199 medium with $1 \% \mathrm{FBS}$, although the other components remained unchanged. When the cells had been cultured in a $37^{\circ} \mathrm{C}$ incubator for $10-15 \mathrm{~h}$, $90 \mu \mathrm{l}$ fresh M199 culture medium with $16 \%$ FBS was added. On the following day (within $24 \mathrm{~h}$ ), D-glucose was added to the normal culture medium to obtain a final concentration of $30.5 \mathrm{mmol} / \mathrm{l}$. The medium from the cells cultured with normal medium was changed every other day. The cells were incubated in a $37^{\circ} \mathrm{C}$ and $5 \% \mathrm{CO}_{2}$ cell culture incubator. At $4 \mathrm{~h}$ before the end of culture, this medium was removed and $100 \mu \mathrm{l} /$ well fresh M199 medium with 10\% FBS and $10 \mu \mathrm{l} /$ well alamarBlue ${ }^{\circledR}$ solution were added. The alamarBlue ${ }^{\circledR}$ cell proliferation assay was performed according to the kit manufacturer's instructions (Merck \& Co., White House Station, NJ, USA). The cells were incubated for $4 \mathrm{~h}$ at $37^{\circ} \mathrm{C}$. The 570 and $600 \mathrm{~nm}$ absorbance optical density values of each well were examined with a microplate reader. The alamarBlue ${ }^{\circledR}$ reduction rates were calculated according to the manufacturer's instructions. Five repeated wells were used at each time point.

Analysis of HUVEC apoptosis. The HUVEC cells were pretreated with quercetin, LY294002 or U0126(Sigma-Aldrich) for $45 \mathrm{~min}$ before $\mathrm{HG}$ was added. At $48 \mathrm{~h}$ after treatment, $1-5 \times 10^{5}$ cells were collected using trypsin without EDTA. Following digestion, the cells were maintained in culture medium with serum to prevent further trypsin digestion. The cells were then washed twice in PBS, with centrifugation at $155 \mathrm{x} \mathrm{g}$ for $5 \mathrm{~min}$, and resuspended in $500 \mu \mathrm{l}$ binding buffer. Subsequently, $5 \mu$ l Annexin V-fluorescein isothiocyanate (FITC; Sigma-Aldrich) and $5 \mu \mathrm{l}$ propidium iodide (PI; Biyuntian, Hangzhou, China) were added and the solutions were mixed. The mixture was incubated at room temperature for 10-15 min in a cool dark place. Annexin V-FITC binding was analyzed by flow cytometry $(E x=488 \mathrm{~nm}$; Em=530 nm; Beckman Coulter, Carlsbad, CA, USA) using a FITC signal detector (usually FL1) and PI staining was examined using a phycoerythrin emission signal detector (usually FL2). Experiments were conducted in six-well plates and were repeated three times.

Western blotting. The HUVEC cells were pretreated with LY294002 $(0.1,1$ or $10 \mu \mathrm{M})$ or $\mathrm{U} 0126(0.1,1$ or $10 \mu \mathrm{M})$ for $45 \mathrm{~min}$ before the addition of HG. Following treatment, HUVEC cells were washed with ice-cold PBS and then cellular proteins were obtained. Equal quantities of protein were then separated by $12 \%$ SDS-PAGE and transferred to

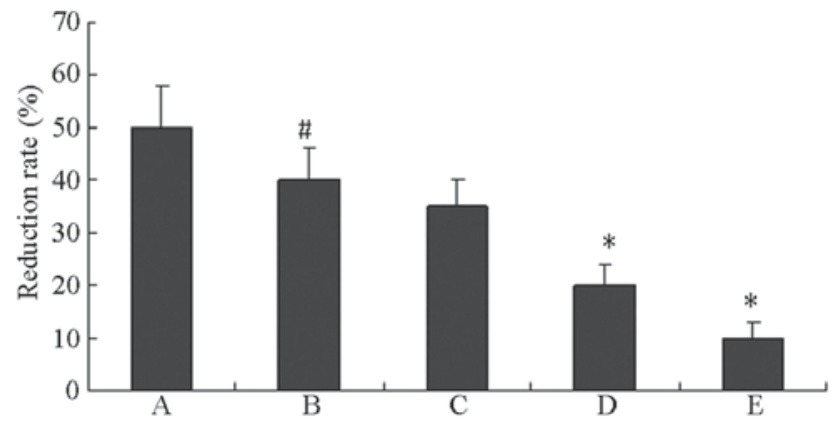

Figure 1. Effect of quercetin on the proliferation of human umbilical vein endothelial cells in response to high glucose $(30.5 \mathrm{mmol} / \mathrm{l})$. Bars: A, control; B, high glucose; C-E, quercetin $(1,10$ and $100 \mu \mathrm{mol} / \mathrm{l})+$ high glucose. ${ }^{\#} \mathrm{P}<0.01$ vs the control group; ${ }^{*} \mathrm{P}<0.01$ vs. the high glucose group.

polyvinylidene difluoride membranes, which were incubated at room temperature in Tris-buffered saline and Tween 20 (TBST) containing 5\% bovine serum albumin. Subsequent to blocking, the membranes were incubated with primary rabbit-anti-human phosphorylated-(p-)HSP27 antibody (1:300; Bioworld Technology, Inc., Minneapolis, MN, USA) and rat-anti-human total-HSP27 antibody (1:500; Santa Cruz Biotechnology, Inc., Santa Cruz, CA, USA) overnight at $4^{\circ} \mathrm{C}$, followed by three washes (10 min each) with TBST. The membranes were then incubated with secondary antibody (goat anti-rabbit for p-HSP27 and goat anti-rat for total-HSP27; Biyuntian) at a dilution of 1:5,000 at room temperature for $70 \mathrm{~min}$, followed by washing three times (10 min each) with TBST. The signals were detected using a chemiluminescence kit (Biyuntian) and quantified by densitometric analysis. Experiments were conducted in six-well plates and were repeated three times.

Statistical analysis. Statistical analysis was conducted using analysis of variance with SPSS 11.5 software (SPSS, Inc., Chicago, IL, USA). Values are expressed as the mean \pm standard deviation.

\section{Results}

Effect of quercetin on HUVEC proliferation induced by $H G$. As shown in Fig. 1, compared with the normal controls, the HUVECs grown in HG exhibited significantly lower cell proliferation $(\mathrm{P}<0.01)$. The proliferation of HUVECs in $\mathrm{HG}$ was reduced by the specific HSP27 inhibitor quercetin in a concentration-dependent manner.

Effect of quercetin on the apoptosis of HUVECs induced by $H G$. As shown in Fig. 2, HG significantly promoted the apoptosis of HUVECs compared with the control $(\mathrm{P}<0.01)$. The apoptosis of HUVECs in HG was further induced by quercetin in a concentration-dependent manner.

Effect of LY294002 and U0126 on HSP27 phosphorylation in HUVECs induced by HG. HSP27 phosphorylation induced by HG was blocked by the specific PI3K inhibitor LY294002 and the specific ERK1/2 inhibitor U0126 in a concentration-dependent manner. As shown in Figs. 3 and 4, 


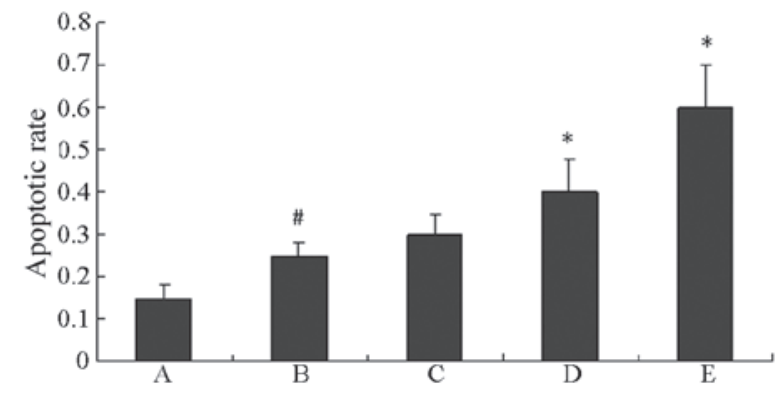

Figure 2. Effects of quercetin on the apoptosis of human umbilical vein endothelial cells in response to high glucose $(30.5 \mathrm{mmol} / \mathrm{l})$. Bars: A, control; B, high glucose; C-E, quercetin $(1,10$ and $100 \mu \mathrm{mol} / \mathrm{l})+$ high glucose.

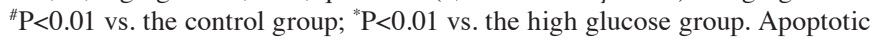
rate $=$ apoptotic cells/total cells.
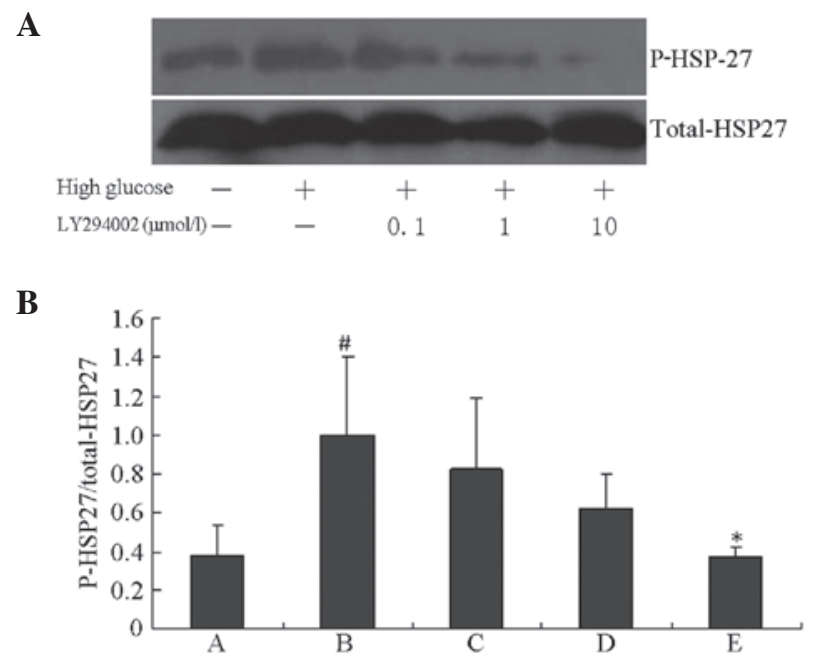

Figure 3. Concentration-dependent inhibitory effects of LY294002 (0.1-10 $\mu \mathrm{mol} / 1)$ on HSP27 phosphorylation induced by high glucose $(30.5 \mathrm{mmol} / \mathrm{l})$ in human umbilical vein endothelial cells. Bars: A, control; $\mathrm{B}$, high glucose; C-E, LY294002 (0.1-10 $\mu \mathrm{mol} / \mathrm{l})+$ high glucose. ${ }^{\#} \mathrm{P}<0.01 \mathrm{vs}$. the control group; ${ }^{*} \mathrm{P}<0.01$ vs. the high glucose group. HSP27, heat shock protein 27; P-, phosphorylated-.

A

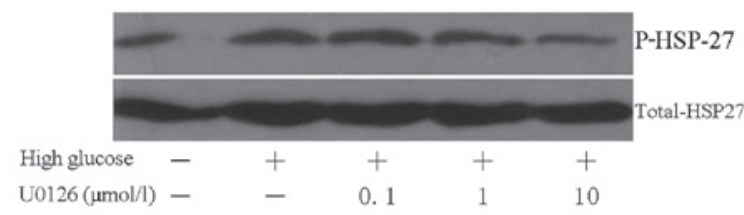

B

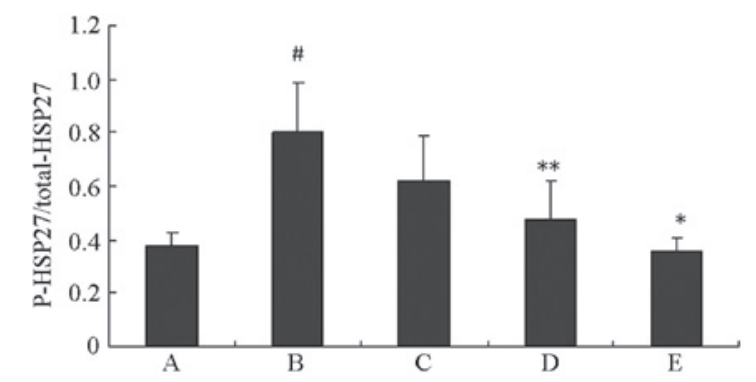

Figure 4. Concentration-dependent inhibitory effects of U0126 (0.1-10 $\mu \mathrm{mol} / \mathrm{l})$ on HSP27 phosphorylation induced by high glucose $(30.5 \mathrm{mmol} / \mathrm{l})$ in human umbilical vein endothelial cells. Bars: A, control; B, high glucose; C-E, U0126 (0.1-10 $\mu \mathrm{mol} / \mathrm{l})+$ high glucose. ${ }^{~} \mathrm{P}<0.01 \mathrm{vs}$. the control group; ${ }^{* *} \mathrm{P}<0.05$ and ${ }^{*} \mathrm{P}<0.01$ vs. the high glucose group. HSP27, heat shock protein 27; P-, phosphorylated-.

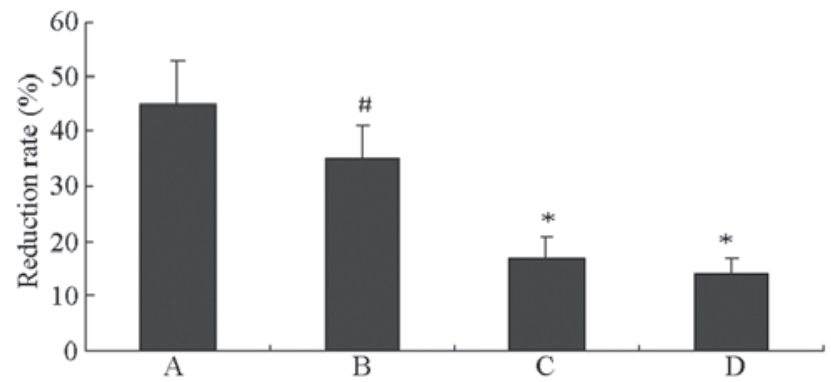

Figure 5. Effects of LY294002 $(10 \mu \mathrm{mol} / 1)$ and U0126 $(10 \mu \mathrm{mol} / 1)$ on the proliferation of human umbilical vein endothelial cells in response to high glucose (30.5 mmol/l). Bars: A, control; B, high glucose; C, LY294002 + high glucose; D, U0126 + high glucose. ${ }^{\#} \mathrm{P}<0.01$ vs. the control group; ${ }^{*} \mathrm{P}<0.01$ vs. the high glucose group.

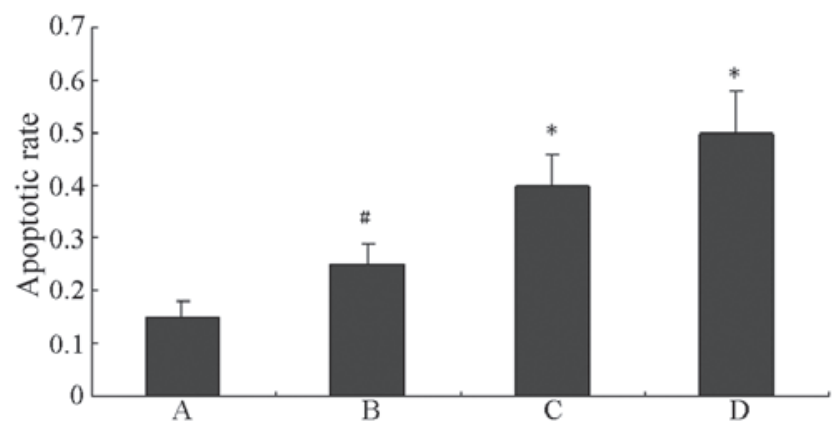

Figure 6. Effects of LY294002 (10 $\mu \mathrm{mol} / \mathrm{l})$ and U0126 $(10 \mu \mathrm{mol} / \mathrm{l})$ on the apoptosis of human umbilical vein endothelial cells in response to high glucose (30.5 mmol/1). Bars: A, control; B, high glucose; C, LY294002 + high glucose; D, U0126 + high glucose. ${ }^{\#} \mathrm{P}<0.01$ vs. the control group; ${ }^{*} \mathrm{P}<0.01$ vs. the high glucose group. Apoptotic rate $=$ apoptotic cells/total cells .

HG induced significant increases in HSP27 phosphorylation compared with the control group $(\mathrm{P}<0.01)$. The peak inhibition rates of HSP27 phosphorylation induced by HG were 62.6 and 56.1\% following $10 \mu \mathrm{M}$ LY294002 and U0126 treatments, respectively ( $\mathrm{P}<0.01$ vs. the $\mathrm{HG}$ groups).

Effect of LY294002 and U0126 on HUVEC proliferation induced by HG. HUVECs grown in HG exhibited significantly inhibited cell proliferation compared with the normal control cells $(\mathrm{P}<0.01)$. The proliferation of HUVECs in HG was further inhibited by LY294002 and U0126, with peak inhibition rates of 51.4 and $60.0 \%$, respectively $(\mathrm{P}<0.01$ vs. the $\mathrm{HG}$ groups; Fig. 5).

Effect of LY294002 and U0126 on HUVEC apoptosis induced by $H G$. An incubation time period of $48 \mathrm{~h}$ was selected as an intervention time point and flow cytometry was used to detect HUVEC apoptosis. As shown in Fig. 6, compared with the normal control group, the HG group exhibited a significant increase in cell apoptosis $(\mathrm{P}<0.01)$. HUVEC apoptosis following $\mathrm{HG}$ was further increased by treatment with either LY294002 or U0126 ( $\mathrm{P}<0.01$ vs. the HG groups).

\section{Discussion}

Although HG has been reported to inhibit EC proliferation and increase apoptosis, the exact mechanism remains largely 
unknown. HSP27, with a molecular weight of $27 \mathrm{kDa}$, has been shown to form large aggregates of $\leq 800 \mathrm{kDa}$ in the cell cytosol. HSP27 expression is upregulated during the stress response and correlates with increased survival ability in cells exposed to cytotoxic stimuli. HSP27 has been shown to prevent the cell death caused by a variety of toxic agents that promote apoptosis. HSP27 is regulated by means of phosphorylation and dephosphorylation. Studies have shown that HSP27 is important in the regulation of proliferation and apoptosis in a number of cell types. HG is known to activate HSP27; however, since multiple signaling pathways are activated during HG-induced EC injury, the role of HSP27 activation in this process requires further clarification. Thus, in the present study, the effect of quercetin (the specific HSP27 inhibitor) on the proliferation and apoptosis of HUVECs induced by HG was examined.

A novel method for measuring cell proliferation, which uses alamarBlue ${ }^{\circledR}$, has recently become available. alamarBlue ${ }^{\circledR}$ is a safe, nontoxic aqueous dye that is used to assess cell viability and cell proliferation. In the present study, the results of the alamarBlue ${ }^{\circledR}$ assay demonstrated the apparent toxic effects of HG on HUVECs and suggested that the specific HSP27 inhibitor quercetin may enhance the inhibition of HUVEC proliferation by HG.

Currently, apoptosis of ECs is considered to be an important cause of atherosclerosis during the development of diabetic chronic vascular diseases. A number of studies have observed that HG increases the rate of apoptosis in cultured HUVECs. The results of the present study revealed that co-administration of quercetin promoted the apoptosis of cultured HUVECs to a greater extent than HG alone, suggesting that HSP27 phosphorylation protects HUVECs from apoptosis. However, the underlying mechanism for this remains unclear. Long-term exposure to HG levels may induce several metabolic changes in ECs; these include negative feedback mechanisms that regulate upstream regulatory factors, which counteracts certain effects of glucose toxicity. This benefits the growth of ECs in HG culture. The present study demonstrated that the proliferation and apoptosis of HUVECs induced by HG was affected by quercetin in a concentration-dependent manner. Therefore, HSP27 phosphorylation may be part of an important signaling pathway that contributes to HUVEC proliferation and apoptosis induced by HG. Subsequently, the signal transduction pathways associated with HSP27 phosphorylation in HUVECs induced by HG were analyzed.

PI3K and Akt are downstream effectors of insulin signaling, and are important in ECs, as these signaling pathways regulate angiogenesis and proliferation (18). In ECs, Akt activation has been reported to promote cell survival $(19,20)$. Studies have shown that the PI3K/Akt/nitric oxide signaling pathway is important in preventing reactive oxygen species-induced EC injury $(21,22)$. However, whether the PI3K/Akt signaling pathway regulates the cell apoptosis induced by $\mathrm{HG}$ remains unclear. MAPK/ERK is an important signaling pathway involved in the regulation of various cellular processes, mainly proliferation and survival, but also apoptosis under particular pathological conditions (23). The MAPK/ERK signaling pathway is mainly involved in mediating growth factor-stimulated processes, such as division, growth, differentiation and survival in various cell types, including HUVECs.
The present study demonstrated that the HSP27 phosphorylation and cell proliferation induced by HG was blocked by the specific PI3K and ERK1/2 inhibitors LY294002 and U0126, and that the apoptosis of HUVECs in the LY294002 + HG and U0126 + HG groups was increased compared with the HG-only group. Therefore, PI3K/Akt and ERK1/2 may be important signaling pathways that contribute to HSP27 phosphorylation, and the proliferation and apoptosis of HUVECs in response to $\mathrm{HG}$. In conclusion, the inhibition of the PI3K/Akt and ERK1/2 signal transduction pathways was found to contribute to the suppression of HSP27 phosphorylation and increased cell apoptosis. As determined by these findings, these two signaling pathways may be involved in the phosphorylation of HSP27 and HUVEC apoptosis in response to HG. Therefore, upregulation of the HSP27 signaling pathway may be considered as a target in future therapies protecting the diabetic patient from macrovascular complications. The present study provides further evidence that HSP27 functions within an anti-apoptotic signaling pathway and may indicate an alternative therapeutic method in the treatment of diabetic cardiovascular complications $(24,25)$.

\section{Acknowledgements}

This study was supported by the Natural Science Foundation (grant no. 2013J01272) and the Key Clinical Speciality Discipline Construction Program of Fujian, China.

\section{References}

1. Chen G, Shen X, Yao J, et al: Ablation of NF-kappaB expression by small interference RNA prevents the dysfunction of human umbilical vein endothelial cells induced by high glucose. Endocrine 35: 63-74, 2009.

2. Chen G, Chen YF, Chen HF, et al: The effect of NF- $\mathrm{kB}$ pathway on proliferation and apoptosis of human umbilical vein endothelial cells induced by intermittent high glucose. Mol Cell Biochem 347: 127-133, 2011.

3. Schisano B, Harte AL, Lois K, et al: GLP-1 analogue, Liraglutide protects human umbilical vein endothelial cells against high glucose induced endoplasmic reticulum stress. Regul Pept 174: 46-52, 2012.

4. Sun F, Zhou B, Lin X and Duan L: Proteomic analysis identifies nuclear protein effectors in PKC-delta signaling under high glucose-inducedapoptosis in human umbilical vein endothelial cells. Mol Med Rep 4: 865-872, 2011.

5. Son TW, Yun SP, Yong MS, et al: Netrin-1 protects hypoxia-induced mitochondrial apoptosis through HSP27 expression via DCC- and integrin $\alpha 6 \beta 4$-dependent Akt, GSK-3 $\beta$, and HSF-1 in mesenchymal stem cells. Cell Death Dis 4: e563, 2013.

6. Arany I, Clark JS, Reed DK, Ember I and Juncos LA: Cisplatin enhances interaction between p66Shc and HSP27: its role in reorganization of the actin cytoskeleton in renal proximal tubule cells. Anticancer Res 32: 4759-4763, 2012.

7. Grogan PT, Sleder KD, Samadi AK, et al: Cytotoxicity of withaferin A in glioblastomas involves induction of an oxidative stress-mediated heat shock response while altering Akt/mTOR and MAPK signaling pathways. Invest New Drugs 31: 545-557, 2013.

8. McClung HM, Golembieski WA, Schultz CR, et al: Deletion of the SPARC acidic domain or EGF-like module reduces SPARC-induced migration and signaling through p38 MAPKHSP27 in glioma. Carcinogenesis 33: 275-284, 2012.

9. Meng G, Sun Y, Fu W, Guo Z and Xu L: Microcystin-LR induces cytoskeleton system reorganization through hyperphosphorylation of tau and HSP27 via PP2A inhibition and subsequent activation of the p38 MAPK signaling pathway in neuroendocrine (PC12) cells. Toxicology 290: 218-229, 2011. 
10. Kim J, Kang D, Sun BK, Kim JH and Song JJ: TRAIL/MEKK4/p38/HSP27/Akt survival network is biphasically modulated by the Src/CIN85/c-Cbl complex. Cell Signal 125 372-379, 2013.

11. Ghosh A, Lai C, McDonald S, et al: HSP27 expression in primary colorectal cancers is dependent on mutation of KRAS and PI3K/AKT activation status and is independent of TP53. Exp Mol Pathol 94: 103-108, 2013.

12. Bhargavan B, Fatma N, Chhunchha B, et al: LEDGF gene silencing impairs the tumorigenicity of prostate cancer DU145 cells by abating the expression of Hsp27 and activation of the Akt/ERK signaling pathway. Cell Death Dis 3: e316, 2012.

13. Qi S, Xin Y, Qi Z, et al: HSP27 phosphorylation modulates TRAIL-induced activation of Src-Akt/ERK signaling through interaction with $\beta$-arrestin2. Cell Signal 26: 594-602, 2014.

14. Kim J, Kim SY, Kang S, et al: HSP27 modulates survival signaling networks in cells treated with curcumin and TRAIL. Cell Signal 24: 1444-1452, 2012.

15. Chen NG, Lu CC, Lin YH, et al: Proteomic approaches to study epigallocatechin gallate-provoked apoptosis of TSGH-8301 human urinary bladder carcinoma cells: roles of AKT and heat shock protein 27 -modulated intrinsic apoptotic pathways. Oncol Rep 26: 939-947, 2011.

16. Chen HF, Xie LD and Xu CS: Role of heat shock protein 27 phosphorylation in migration of vascular smooth muscle cells Mol Cell Biochem 327: 1-6, 2009.

17. Chen HF, Xie LD and Xu CS: The signal transduction pathways of heat shock protein 27 phosphorylation in vascular smooth muscle cells. Mol Cell Biochem 333: 49-56, 2010.

18. Ueda K, Nakahara T, Akanuma K, et al: Differential effects of LY294002 and wortmannin on neurons and vascular endothelial cells in the rat retina. Pharmacol Rep 65: 854-862, 2013.
19. You JJ, Yang CH, Yang CM and Chen MS: Cyr61 induces the expression of monocyte chemoattractant protein-1 via the integrin $\alpha v \beta 3$, FAK, PI3K/Akt, and NF- $\mathrm{BB}$ pathways in retinal vascular endothelial cells. Cell Signal 26: 133-140, 2014

20. Chen ML, Lin YH, Yang CM and Hu ML: Lycopene inhibits angiogenesis both in vitro and in vivo by inhibiting MMP-2/uPA system through VEGFR2-mediated PI3K-Akt and ERK/p38 signaling pathways. Mol Nutr Food Res 56: 889-899, 2012

21. Fournier NM, Lee B, Banasr M, Elsayed M and Duman RS: Vascular endothelial growth factor regulates adult hippocampal cell proliferation through MEK/ERK- and PI3K/Akt-dependent signaling. Neuropharmacology 63: 642-652, 2012.

22. Oviedo-Boyso J, Cortés-Vieyra R, Huante-Mendoza A, et al: The phosphoinositide-3-kinase-Akt signaling pathway is important for Staphylococcus aureus internalization by endothelial cells. Infect Immun 79: 4569-4577, 2011.

23. Shaul YD and Seger R: The MEK/ERK cascade: from signaling specificity to diverse functions. Biochim Biophys Acta 1773: 1213-1226, 2007.

24. Will M, Qin AC, Toy W, et al: Rapid induction of apoptosis by PI3K inhibitors is dependent upon their transient inhibition of RAS-ERK signaling. Cancer Discov 4:334-347, 2014.

25. Wakao K, Watanabe T, Takadama T, et al: Sangivamycin induces apoptosis by suppressing Erk signaling in primary effusion lymphoma cells. Biochem Biophys Res Commun 444:135-140, 2014. 